Aim To evaluate current practice in requesting salicylate levels in children presenting with intentional overdose.

Background Intentional overdose is a common presentation in children's emergency departments but the pattern of medications taken has changed over time. Over the last two decades use of aspirin (salicylate) has markedly reduced since recognition of Reye's Syndrome in children. Toxbase and NPIS advise salicylate levels are not required unless there is admission of ingestion, symptoms of salicylate toxicity or in the unconscious patient. It was noted that it remains common practice to check salicylate levels in children with intentional overdose.

Methods A retrospective review of all children presenting with intentional overdose at a busy two site acute trust. Case note and blood results review of all children coded as poisoning between July - December 2012, excluding children with accidental overdose.

Results There were 59 episodes of intentional overdose with 53 females and 6 males. The average age was 14yrs (11 - 16yrs). $73 \%$ (43) of the patients were asymptomatic. The most common drug taken was paracetamol (46\%), followed by ibuprofen (20\%) and cocodamol (11\%). Only 2 patients (3\%) admitted taking salicylate. The majority of initial blood tests were taken in the emergency department (71\%). 83\% (49) had salicylate levels measured and only 4\% (2) had abnormal levels. Both of these patients had admitted salicylate ingestion. No patients who denied salicylate ingestion were found to have raised levels. Salicylate levels were deemed appropriate and useful in $4(8 \%)$ patients due to history or symptoms. It was also observed that there was frequent requesting of a wide range of unnecessary blood tests including CK, FBC and CRP.
Conclusion There is considerable over testing of salicylate levels in children with intentional overdose. Our results found the test appropriate in only $4(8 \%)$ patients. The laboratory test itself is relatively cheap however, in combination with other unnecessary tests, the cumulative cost is significant. We advise improved education as to the indications for salicylate levels as part of aiming to reduce unnecessary investigations and costs in children's emergency department.

\section{G92(P) PAEDIATRIC CARDIAC MURMUR GUIDELINE FOR THE CHILDREN'S EMERGENCY DEPARTMENT}

P Venugopalan, M Ranaweera. Paediatrics, Brighton and Sussex University Hospitals, Brighton, UK

\subsection{6/archdischild-2015-308599.91}

Aim To develop a paediatric cardiac murmur guideline for a Children's emergency department (CED), following mismanagement of 2 cases of heart murmurs in children. Currently there is no national guideline in place.

Methods Electronic databases Medline, Embase, Cochrane Cinahl, Psycinfo, Amed, British nursing index, DARE and CENTRAL were searched using title/abstract terms and subject headings: heart/cardio/cardia/murmur/sound AND diagnosis/ assessment/evaluation/examination. Results were limited to children aged 0-18 years and no limitations placed on date or language. The results were exported on to Mendeley workstation and analysed.

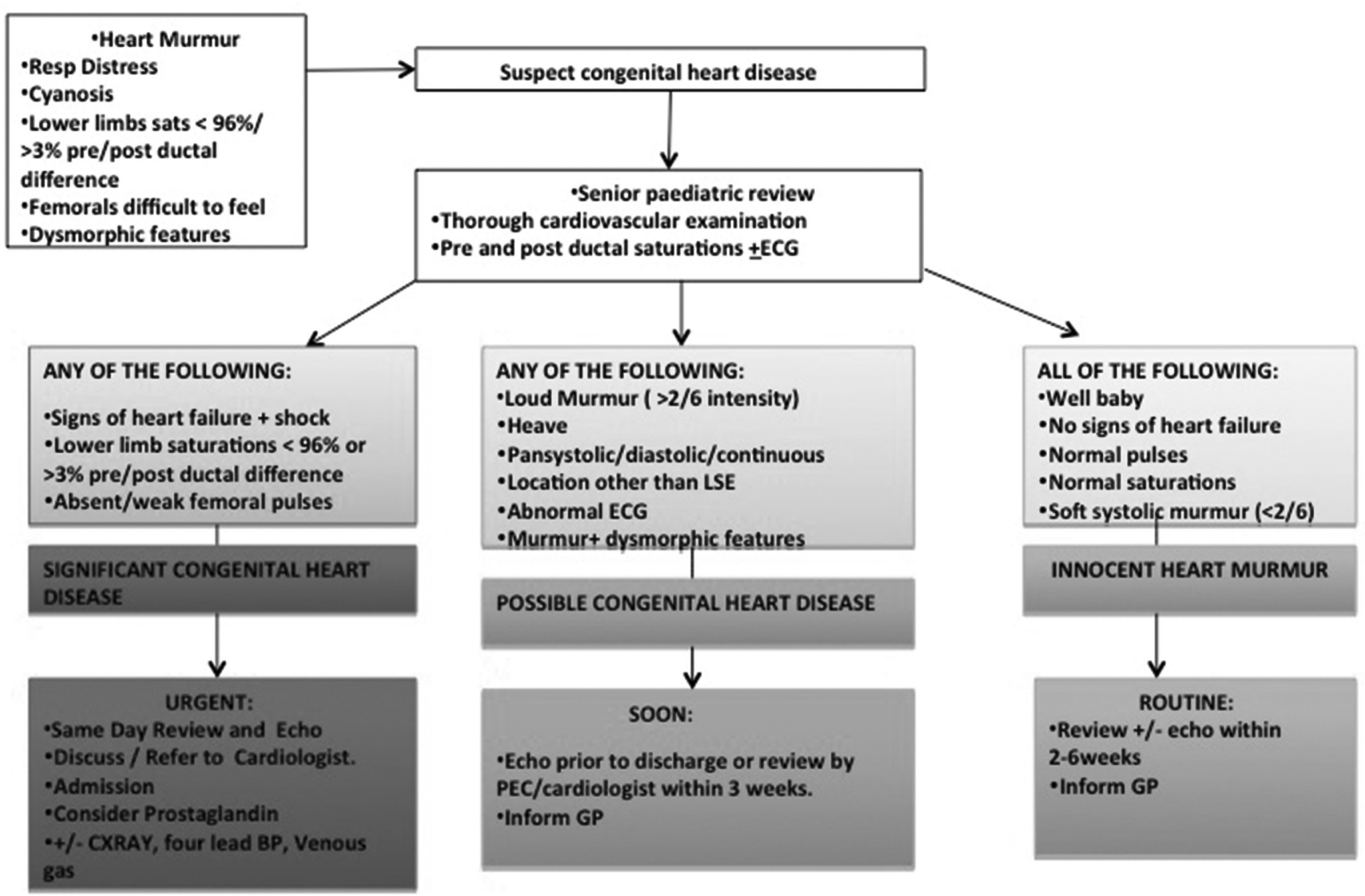

Abstract G92(P) Figure 1 Neonatal murmur algorithm 


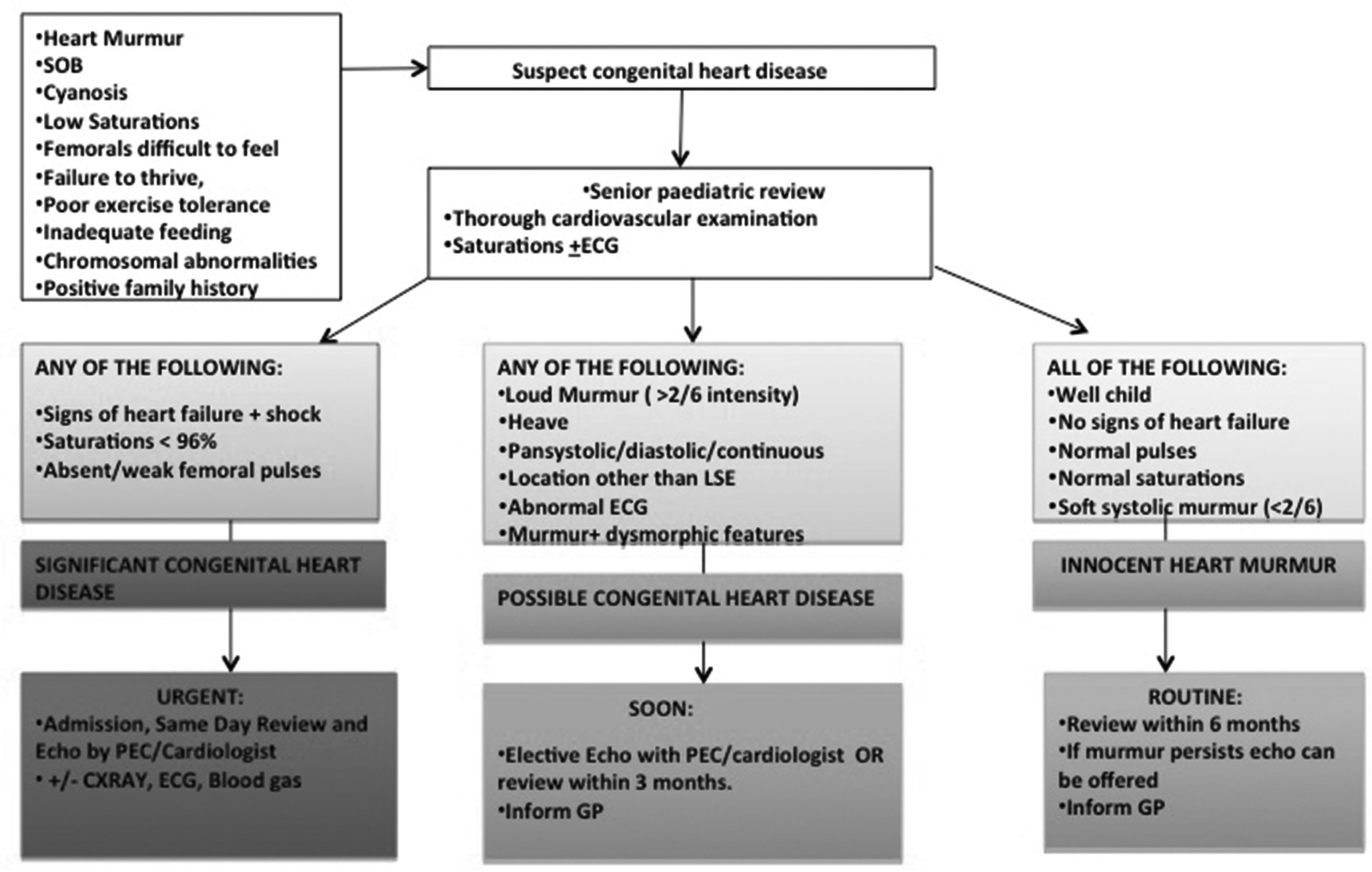

Abstract G92(P) Figure 2 Older children murmur algorithm

Results Medline returned 1204 results, Embase (1168), Cochrane (0), Cinahl (131), Psycinfo (15), Amed (13), British nursing index (5), DARE (0) and CENTRAL (8). When duplicates were removed 2533 results remained from which 560 articles were selected according to suitability. Among these 100 original papers were fully accessed and reviewed. Based on the available evidence a guideline triaging patients into a traffic-light system according to clinical severity with an algorithm for investigation was developed, taking into account patient feedback and opinions from experts in the field (Figures 1 and 2).

Conclusion A new, easy to follow guideline has been implemented, which will aid clinicians to confidently triage children presenting with murmurs according to clinical need and guide appropriate management. This will promote quality of care and patient safety, and facilitate the management of children with murmurs who attend the CED.

\section{G93(P) DO AS I SAY, NOT AS I DO? DIFFERENCES BETWEEN PERCEIVED AND ACTUAL PRACTICE IN THE FOLLOW UP OF MICROSCOPIC HAEMATURIA/PROTEINURIA IN FEBRILE CHILDREN SEEN IN THE CHILDREN'S EMERGENCY DEPARTMENT}

${ }^{1}$ RA Hastings, ${ }^{2} \mathrm{AJ}$ Lunn, ${ }^{3} \mathrm{~J}$ Surridge. 'Department of Paediatrics, Nottingham Children's Hospital, Nottingham, UK; ${ }^{2}$ Children's Renal and Urology Unit, Nottingham Children's Hospital, Nottingham, UK; ${ }^{3}$ Children's Emergency Department, Derbyshire Children's Hospital, Derby, UK

\subsection{6/archdischild-2015-308599.92}

Aims To examine the follow up of children with microscopic haematuria/proteinuria in the children's emergency department
(CED) and to see if it differs from the perceived practice of CED doctors.

Methods An audit of children with a diagnosis of 'viral URTI' or 'viral illness' during one month in a busy ( $>30,000$ attendances/year) CED was performed. The follow up of those children with a recorded positive urine dipstick was examined.

A survey of responses to scenarios of children with an incidental finding of microscopic haematuria/proteinuria (with no overt signs of renal disease) was used. The survey was sent to SHO grade doctors and registrars and asked for their current practice in arranging follow up of children with positive urine dipstick results.

Results 174 children were audited with 24 of them having a documented positive urine dipstick result. Seventeen children had a single positive (either haematuria/proteinuria), none had follow up. Seven children had at least $2+$ of either proteinuria or haematuria and only one of these had any form of follow up arranged (14\%).

Online survey of SHO doctors (8) and registrars (12) showed that for a child with a $1+$ positive urine dip only 5 doctors (25\%) would arrange follow up. However 17 doctors (85\%) said they routinely offer follow up to children with at least $2+$ on urine dip (either haematuria or proteinuria).

Conclusions Approximately 2/3 of microscopic haematuria/proteinuria in children without specific renal symptoms resolves. Ensuring resolution is important since up to $50 \%$ of children in whom it persists have renal disease. ${ }^{1}$ In our CED, only $14 \%$ of children with at least $2+$ positive urine dipstick had follow up arranged.

On survey, $85 \%$ of doctors working in CED stated that their current practice is to offer follow up to these same children. In regards to the management of haematuria/proteinuria, doctors' 